\title{
Dark energy and the refined de sitter conjecture
}

\author{
Prateek Agrawal and Georges Obied \\ Jefferson Physical Laboratory, Harvard University, \\ 17 Oxford Street, Cambridge, MA 02138, U.S.A. \\ E-mail: prateekagrawal@fas.harvard.edu, gobied@g.harvard.edu
}

ABSTRACT: We revisit the phenomenology of quintessence models in light of the recently refined version of the de Sitter Swampland conjecture, which includes the possibility of unstable de Sitter critical points. We show that models of quintessence can evade previously derived lower bounds on $(1+w)$, albeit with very finely-tuned initial conditions. In the absence of such tuning or other rolling quintessence fields, a field with mass close to Hubble is required, which has a generic prediction for $(1+w)$. Slow-roll single field inflation models remain in tension. Other phenomenological constraints arising from the coupling of the quintessence field with the Higgs or the QCD axion are significantly relaxed.

Keywords: Cosmology of Theories beyond the SM, Effective Field Theories

ARXIV EPRINT: 1811.00554 


\section{Contents}

1 Introduction 1

2 Elsewhere on the moduli space $\quad 2$

3 Dark energy 3

4 Conclusion and discussion $\quad 7$

\section{Introduction}

A class of effective field theories (EFTs), while otherwise consistent, do not admit a UVcompletion within a theory of quantum gravity. Such EFTs are said to lie in the Swampland [1]. Delineating the boundaries of the Swampland is an important task that could point to observable consequences of a theory of quantum gravity. Progress has been made by studying the general properties of string compactifications (e.g. see [2-6] or [7] for a recent review).

One recent criterion is the de Sitter conjecture (dSC) which proposes that all scalar potentials must satisfy the condition:

$$
|\nabla V| \geq c \cdot V
$$

where the dimension-dependent constant $c \sim \mathcal{O}(1)$. This condition is motivated by the difficulty of de-Sitter (dS) constructions within string theory and the plethora of no-go theorems which take the form of (1.1) and thus forbid dS spacetimes under restricted circumstances [8-10] (see also [6] and references therein). However, for a different point of view, see for example [11-20].

The dSC has observable consequences for dark energy ${ }^{1}$ equation of state [22] and constrains single field slow-roll inflation. This has led to a number of model-building proposals [23-36] to make inflation consistent with dSC. Similarly, interest in quasi-dS spaces consistent with dSC has motivated the construction of quintessence models [14, 3741]. Beyond these cosmological implications, a strong consequence arises from studying the symmetric point of the Higgs potential [19, 40, 42, 43], forcing the quintessence field to couple with the Higgs boson. A similar conclusion can be derived for the QCD axion [19].

Alternate formulations of the dSC have also been proposed in [42, 44, 45]. We study a particular refinement of the dSC (henceforth referred to as RdSC) which has been proposed in $[46,47]$. The refinement allows $(1.1)$ to be violated if the second derivative of the potential is sufficiently negative. Explicitly, the refined de Sitter conjecture states

$$
|\nabla V| \geq c V \quad \text { or } \quad \min \left(\nabla_{i} \nabla_{j} V\right) \leq-c^{\prime} V
$$

where $c, c^{\prime}$ are constants of $\mathcal{O}(1)$.

\footnotetext{
${ }^{1}$ For a review of quintessence dark energy, see [21].
} 
The RdSC is motivated by its connection to the distance Swampland conjecture [3] through Bousso's covariant entropy bound [48]. The refined conjecture evades all the counter-examples to the dSC $[18,45,49]$ since they involve tachyonic dS critical points [50, 51]. Similarly, it also evades constraints arising from coupling of the quintessence field with the Higgs field and the axion [19, 42].

The original dSC had a firm prediction for the equation of state for the dark energy. In this note we investigate the implications of the refined de Sitter conjecture (RdSC) for dark energy phenomenology. We show that an arbitrarily fine-tuned initial condition can satisfy current and future constraints on $w(z)$ for any values of $c$ and $c^{\prime}$, but generic initial conditions retain a prediction of deviation from $w=-1$.

\section{Elsewhere on the moduli space}

Dark energy observations allow a quintessence field in the current universe with a potential whose slope is of the order of the vacuum energy (in Planck units). Interesting constraints can be derived from considering the potential of the quintessence field along with other scalar fields like the Higgs (and potentially the axion) away from our current position on the moduli space.

If the quintessence does not couple to the Higgs, then the dSC is badly violated at the symmetric point of the Higgs potential [42]. A coupling of the Higgs with the quintessence, on the other hand, leads to larger-than-observed deviations in fifth force experiments, except for perhaps a very fine-tuned set up where this coupling vanishes around the Higgs minimum [19, 42]. More recently it was shown that even this possibility is under tension from time-dependence of the proton-to-electron mass ratio [43].

This tension is relaxed when we consider the refined conjecture. Clearly, at the symmetric point on the Higgs potential the RdSC is satisfied irrespective of the coupling of the Higgs with the quintessence field. However, it is useful to see whether this is true along the entire relevant range of the potential. For illustration we take a toy potential,

$$
V(\phi, h)=\lambda_{h}\left(h^{2}-v^{2}\right)^{2}+V_{0} e^{-\lambda \phi}
$$

For values of $h$ outside a small neighborhood of the origin (and away from the minimum), there is a slope in the $h$ direction which satisfies the slope requirement in (R)dSC,

$$
\frac{M_{\mathrm{Pl}}\left|\nabla_{h} V\right|}{V}>c \Rightarrow h \gtrsim \frac{c v^{2}}{4 M_{\mathrm{Pl}}},
$$

where $c v^{2} / M_{\mathrm{Pl}} \ll v$. However, near the origin, the second derivative $\nabla_{h}^{2} V$ causes the $\mathrm{RdSC}$ to be satisfied. The latter switches sign at $h=v / \sqrt{3}$. So the second derivative is sufficiently negative for $h \sim v / \sqrt{3} \gg v^{2} / M_{\mathrm{Pl}}$,

$$
\frac{M_{\mathrm{Pl}}^{2} \nabla_{h}^{2} V}{V}<-c^{\prime} \Rightarrow h \lesssim \frac{v}{\sqrt{3}}\left[1-\frac{c^{\prime}}{18}\left(\frac{v}{M_{\mathrm{Pl}}}\right)^{2}\right]
$$

Finally, as the $h$ field nears its minimum at $v$, neither of the above conditions is fulfilled, and the (R)dSC is satisfied by the slope in the $\phi$ direction, as long as $\lambda>c$. The norm of 


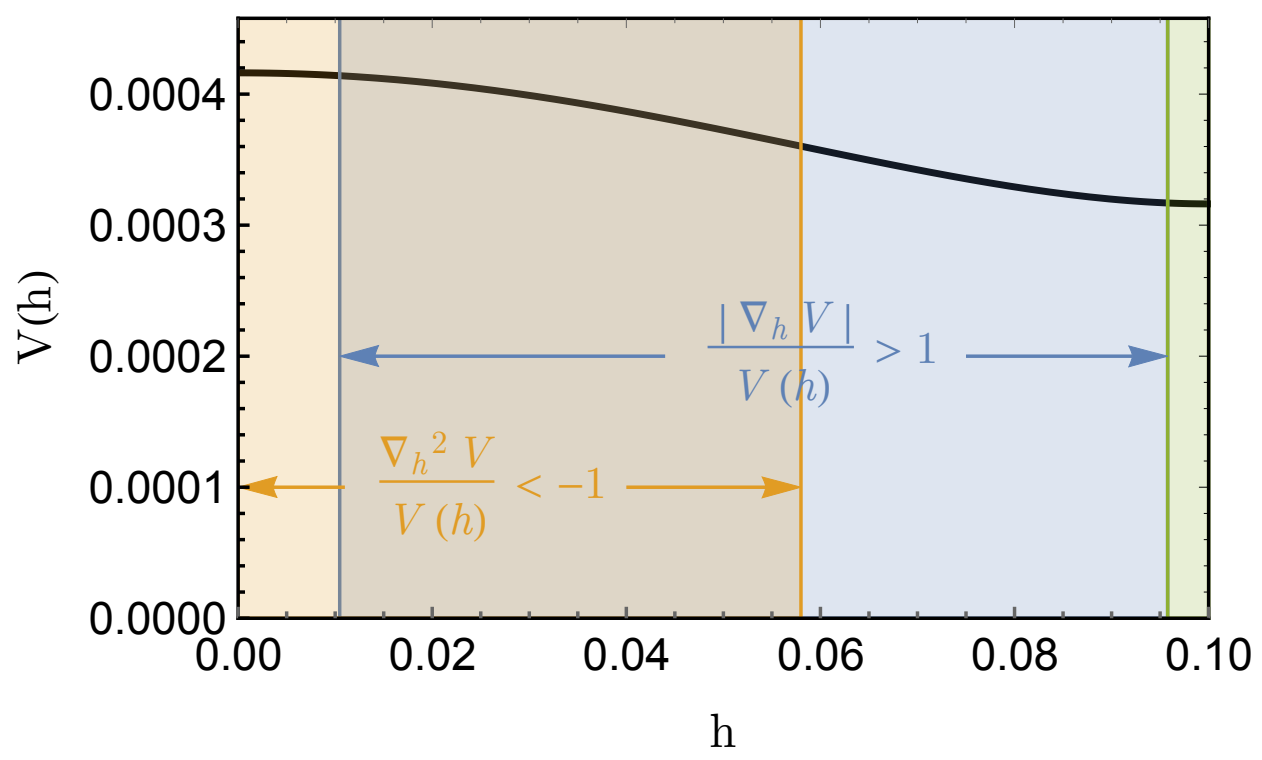

Figure 1. An example scalar potential (e.g. the Higgs potential) with spontaneous symmetry breaking, with parameters exaggerated for clarity. In the blue region, the (R)dSC is satisfied by the $h$ itself. In the orange region, the RdSC is satisfied along the $h$ direction. In the green region near the minimum along the $h$ direction, a "quintessence" field is needed to satisfy (R)dSC. If (R)dSC is satisfied at the minimum, then it is satisfied in all the green region (see text for details).

the gradient of the potential is sufficiently large even away from the minimum (and well into the region where $\left|\nabla_{h} V\right|$ is large), if the following condition is met for the potential at $h=v$,

$$
V(\phi, v) \lesssim \frac{8 \lambda_{h}}{\lambda^{2}} v^{2} M_{\mathrm{Pl}}^{2}
$$

This is a very mild restriction on the potential. This analysis can be carried out in essentially the same way for the SM Higgs as well as the QCD axion. In figure 1 we show a toy example of this phenomenon. We have chosen exaggerated numerical values for illustration. We see that the RdSC is satisfied everywhere along the potential with no cross coupling requirement between $\phi$ and $h$.

Finally, as noted in [47], the RdSC also resolves issues pointed out by [18] and, in a similar higher dimensional setting, by [19]. The general arguments in these papers only tell us about the presence of an extremum, and in particular a maximum / inflection point. Therefore, the RdSC can be easily consistent with these constructions if the smallest eigenvalue of the Hessian is sufficiently negative at these critical points.

\section{Dark energy}

We now turn to an analysis of the implication of the refinement on dark energy phenomenology. The dSC allows one to place a lower bound on the equation of state parameter $w \equiv p_{\phi} / \rho_{\phi}$ as presented in [22], and using current observations, an upper bound on $c$. We now show that the RdSC allows a class of models where the quintessence field satisfies 


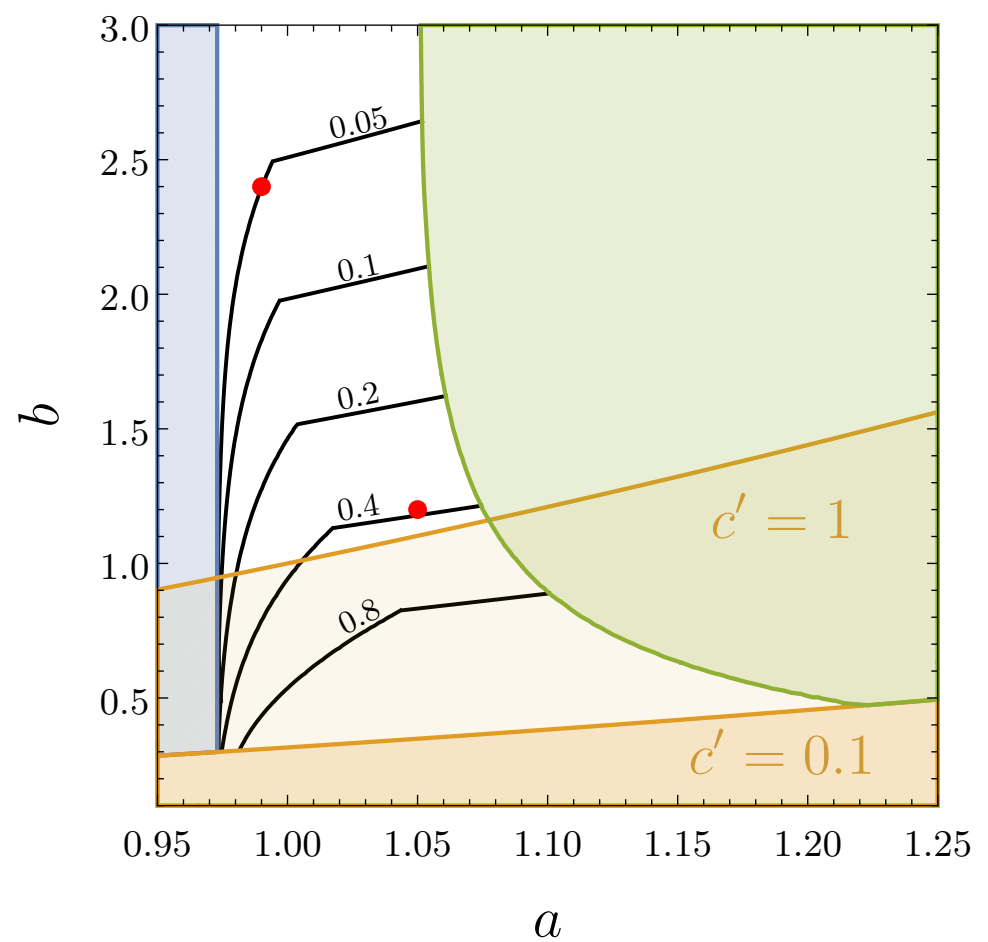

Figure 2. Allowed part of parameter space for our potential (3.1) subject to the RdSC (lower shaded), $w(z)$ and $\Omega_{\Lambda}$ (upper right shaded) and $\Omega_{\Lambda}$ (left shaded) constraints. Contours show the maximum allowed misalignment of the field $\phi / M_{\mathrm{Pl}}$ from the top of the potential. The contour segment to the left of the kink is constrained by $\Omega_{\Lambda}$, whereas the segment to the right is constrained by $w(z)$. Red dots indicate benchmark examples plotted in figures 3 and 4 . We show regions excluded by the RdSC (orange) for $c^{\prime}=0.1$ and $c^{\prime}=1$ for illustration.

the second clause of the refined conjecture, and we cannot put a bound on $c$ using data. Further, one can arrange for $w \approx-1$ today, albeit at the expense of very finely tuned initial conditions. For concreteness we illustrate this with an example potential for the quintessence field of the form,

$$
V(\phi)=V_{0}\left(a^{4}-\frac{1}{2} b^{2} \frac{\phi^{2}}{M_{\mathrm{Pl}}^{2}}\right),
$$

where $V_{0}=(2.23 \mathrm{meV})^{4}$ is the value of dark energy in $\Lambda$ CDM. This potential satisfies the second clause of the RdSC when $b^{2} \geq c^{\prime} a^{4}$. The mass of the field can be conveniently written in terms of the Hubble parameter today, $m_{\phi}^{2}=-b^{2} V_{0} / M_{\mathrm{Pl}}^{2}=-3 b^{2} \Omega_{\Lambda} H_{0}^{2}$, where $\Omega_{\Lambda}=0.692$ is the dark energy fraction of the universe today. Given the dark energy content of the universe today, we see that this potential requires $a \gtrsim 1$. Then RdSC puts a lower bound on the curvature of the quintessence potential, $b^{2}>c^{\prime}$, or $\left|m_{\phi}^{2}\right|>3 c^{\prime} \Omega_{\Lambda} H_{0}^{2}$.

If we ignore quantum fluctuations, then it is clear that we can set the field at the top of the potential, and obtain $w=-1$ exactly. However, quantum fluctuations will destabilize the field from the maximum and fragment it to form domain walls. Therefore, we have to ensure that the field is sufficiently misaligned from the top to behave classically. The 
classical regime only requires a very tiny misalignment,

$$
\phi>H \frac{H^{2}}{3 b^{2} \Omega_{\Lambda} H_{0}^{2}} .
$$

At the same time, if the field has a large misalignment from the maximum, its equation of state will deviate from $w=-1$.

There are two possible allowed regimes. The first is when $b \gg 1$, the slope of the potential is large as soon as the field is misaligned from the maximum, and leads to large deviations in $w+1$. In this case the initial misalignment has to be very finely tuned to be consistent with supernova observations. In the second case, near the boundary of the RdSC constraint, $b^{2} \gtrsim c^{\prime} a^{4}$, the mass of the field is order $H_{0}$, and therefore it only starts rolling today for $\mathcal{O}(1)$ misalignment, making it consistent with observations.

In figure 2 we show the allowed parameter range in $(a, b)$ space. We also show the contours of the maximal initial value of the field that is allowed by observations of $\Omega_{\Lambda}[52]$ and $w(z)$ [53]. As in [22], we choose the $2 \sigma$ contours for $w(z)$ in [53] and for $\Omega_{\Lambda}$ from [54], and find the largest initial misalignment that is consistent with the measurement of $\Omega_{\Lambda}$ and $w(z)$. For $b \gg 1$, we see that the initial values have to be tuned, as noted above. In the region $b \sim 1$, we can allow generic initial conditions. The trajectories of the fields for specific values of $(a, b)$ are shown in figures 3 and 4 . We show the evolution of this system in $(x, y)$ coordinates defined as

$$
x \equiv \frac{\dot{\phi}}{\sqrt{6} M_{\mathrm{Pl}} H} ; \quad y \equiv \frac{\sqrt{V}}{\sqrt{3} M_{\mathrm{Pl}} H},
$$

as well as the evolution of the equation of state $w$. We have chosen examples where the initial conditions are allowed to be generic (figure 3) and where we need to tune the initial conditions to satisfy the supernova constraints (figure 4).

We see that in either case, tuning the initial conditions allows us to push $w$ as close to -1 as we want, evading the lower bound derived in [22]. However, in the absence of this initial condition tuning, a generic prediction on the equation of state can be estimated. If $c^{\prime} \gg 1$, then it is generally hard to satisfy the current $w(z)$ constraints with untuned initial conditions, and the least tuned initial conditions will typically have $(1+w)$ close to the constraints today. If $c^{\prime} \lesssim 1$, then for $b^{2} \sim c^{\prime} a^{4}$ and $a \sim 1$ we can have an $\mathcal{O}\left(M_{\mathrm{Pl}}\right)$ misalignment. The slope of the potential in this case is given by

$$
V^{\prime}(\phi)=V_{0} b^{2} \frac{\phi}{M_{\mathrm{Pl}}} \approx \frac{V_{0}}{M_{\mathrm{Pl}}} c^{\prime}
$$

Using the "slow-roll" approximation, $3 H \dot{\phi} \simeq V^{\prime}(\phi)$, we can estimate the value of $w$ for this parameter choice,

$$
1+w \simeq \frac{\dot{\phi}^{2}}{V_{0}} \sim \frac{V^{\prime 2}}{9 H^{2} V_{0}} \sim \frac{1}{3} \Omega_{\Lambda} c^{\prime 2} .
$$

It is interesting to compare this to a very similar looking bound derived in [22]. We emphasize however that in the current case this is not a hard bound but more a generic 

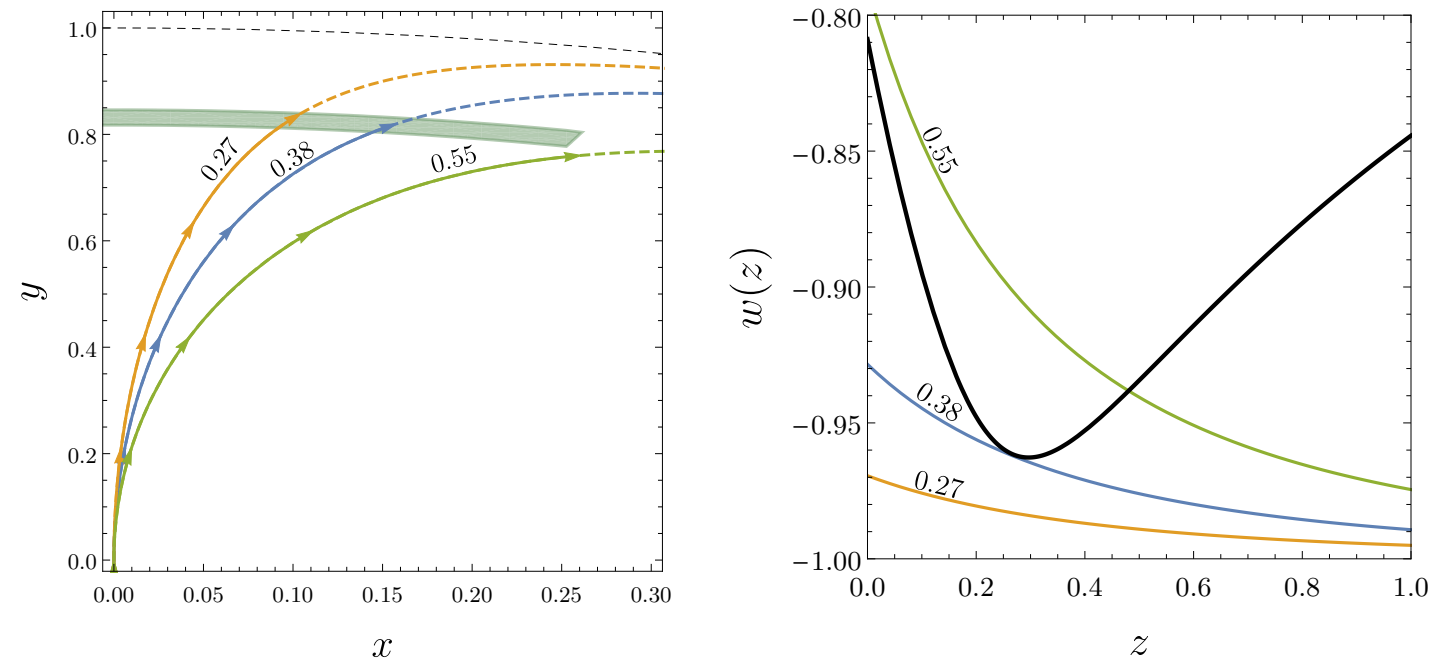

Figure 3. The $(x, y)$ trajectories of the quintessence field (left) and its equation of state (right) for a range of initial misalignment. For this plot, we have chosen the values $(a, b)=(1.05,1.2)$, for which generic initial conditions are consistent with data. Curves are labeled with the initial field value $\phi_{\mathrm{init}} / M_{\mathrm{Pl}}$ and show the minimum and maximum allowed values in addition to a trajectory that is excluded by data.
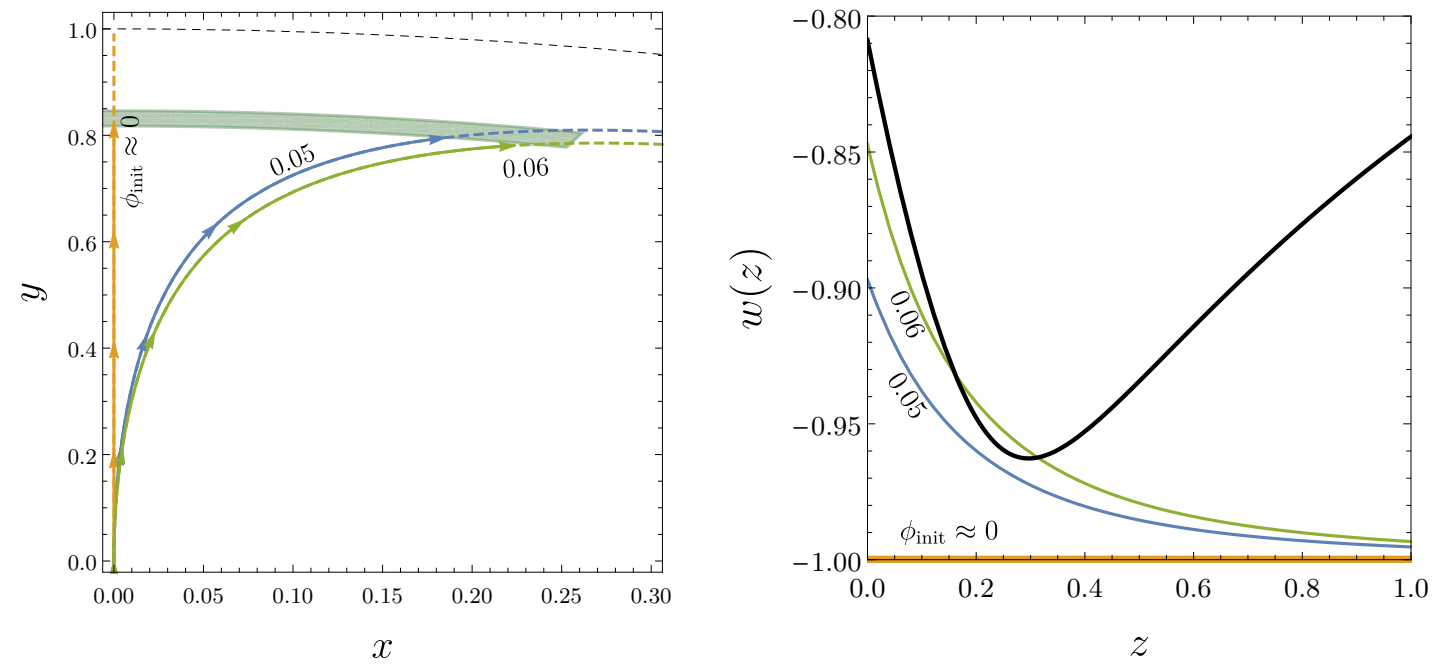

Figure 4. The $(x, y)$ trajectories of the quintessence field (left) and its equation of state (right) for a range of initial misalignment. For this plot, we have chosen the values $(a, b)=(0.99,2.4)$, for which only fine-tuned initial conditions are consistent with data. Curves are labeled with the initial field value $\phi_{\text {init }} / M_{\mathrm{Pl}}$ and show the minimum and maximum allowed values in addition to a trajectory that is excluded by data. 
prediction. Due to this fact, we are unable to derive a robust bound on $c^{\prime}$ using current data as was done for $c$ in [22]. Further, we can trade off bounds on $c$ with bounds on $c^{\prime}$, and with tuned initial conditions we can have both $c$ and $c^{\prime}$ to be $\mathcal{O}(1)$.

\section{Conclusion and discussion}

Swampland conjectures are in a very active phase of exploration. We have studied the implications of the recently proposed refined de Sitter conjecture [44-47]. The conjecture is motivated by an older distance Swampland conjecture and its connection with Bousso's covariant entropy bound. This conjecture circumvents a number of theoretical and phenomenological tensions arising from coupling of the quintessence field to other scalar fields in the standard model and beyond. The RdSC is also consistent with constructions which are claimed to be counter-examples to the earlier de Sitter conjecture.

As in dSC, the RdSC appears to be in tension with single-field slow-roll inflation if both $c, c^{\prime}$ are strictly $\mathcal{O}(1)$ [55]. The fact that either $\left|\epsilon_{V}\right|$ or $\left|\eta_{V}\right|$ are $\mathcal{O}(1)$ makes it impossible to naturally satisfy constraints on the scalar tilt $n_{s}-1 \approx 2 \eta_{V}-6 \epsilon_{V} \approx 0$. In fact, recent analyses [52] show that $\mathcal{O}(1)$ values for either of $\eta_{V}$ or $\epsilon_{V}$ are strongly ruled out. More extended inflationary models can potentially evade this tension; these models often come with detectable deviations from single-field case $[24,27,56]$. Unless the initial conditions are very fine-tuned, the conclusions of [22] for the future cosmology of the universe remain mostly unchanged.

It would be very interesting to try and identify models for such a scalar field. In the string axiverse we expect a plenitude of light scalars, and the positive dark energy of the universe in such a system can be made up of a number of light particles. What the RdSC adds to this picture then is that it hints towards an axion of mass comparable to the Hubble scale, with a misalignment from its minimum of $\mathcal{O}\left(M_{\mathrm{Pl}}\right)$ such that it has just recently begun dominating the universe and appears briefly as dark energy, before eventually starting to oscillate as matter.

\section{Acknowledgments}

We thank David Pinner for useful discussions. We are grateful to Paul Steinhardt and Cumrun Vafa for guidance and useful comments on the manuscript. The work of PA is supported by the NSF grants PHY-0855591 and PHY-1216270.

Open Access. This article is distributed under the terms of the Creative Commons Attribution License (CC-BY 4.0), which permits any use, distribution and reproduction in any medium, provided the original author(s) and source are credited.

\section{References}

[1] C. Vafa, The string landscape and the swampland, hep-th/0509212 [INSPIRE].

[2] T. Banks and N. Seiberg, Symmetries and strings in field theory and gravity, Phys. Rev. D 83 (2011) 084019 [arXiv: 1011.5120] [InSPIRE]. 
[3] H. Ooguri and C. Vafa, On the geometry of the string landscape and the swampland, Nucl. Phys. B 766 (2007) 21 [hep-th/0605264] [INSPIRE].

[4] N. Arkani-Hamed, L. Motl, A. Nicolis and C. Vafa, The string landscape, black holes and gravity as the weakest force, JHEP 06 (2007) 060 [hep-th/0601001] [INSPIRE].

[5] H. Ooguri and C. Vafa, Non-supersymmetric AdS and the swampland, Adv. Theor. Math. Phys. 21 (2017) 1787 [arXiv:1610.01533] [InSPIRE].

[6] G. Obied, H. Ooguri, L. Spodyneiko and C. Vafa, De Sitter Space and the swampland, arXiv: 1806.08362 [INSPIRE].

[7] T.D. Brennan, F. Carta and C. Vafa, The string landscape, the swampland and the missing corner, PoS (TASI2017) 015 [arXiv: 1711.00864] [INSPIRE].

[8] J.M. Maldacena and C. Núñez, Supergravity description of field theories on curved manifolds and a no go theorem, Int. J. Mod. Phys. A 16 (2001) 822 [hep-th/0007018] [INSPIRE].

[9] M.P. Hertzberg, S. Kachru, W. Taylor and M. Tegmark, Inflationary Constraints on Type IIA String Theory, JHEP 12 (2007) 095 [arXiv:0711.2512] [InSPIRE].

[10] T. Wrase and M. Zagermann, On classical de Sitter vacua in string theory, Fortsch. Phys. 58 (2010) 906 [arXiv:1003.0029] [inSPIRE].

[11] E. Silverstein, (A)dS backgrounds from asymmetric orientifolds, Clay Mat. Proc. 1 (2002) 179 [hep-th/0106209] [INSPIRE].

[12] A. Maloney, E. Silverstein and A. Strominger, de Sitter space in noncritical string theory, in the poroceedings of Workshop: The future of theoretical physics and cosmology: Celebrating Stephen Hawking's 60th birthday, January 7-10, Cambridge, U.K. (2002), hep-th/0205316 [INSPIRE].

[13] S. Kachru, R. Kallosh, A.D. Linde and S.P. Trivedi, De Sitter vacua in string theory, Phys. Rev. D 68 (2003) 046005 [hep-th/0301240] [INSPIRE].

[14] M. Cicoli et al., De Sitter vs Quintessence in String Theory, Fortsch. Phys. 67 (2019) 1800079 [arXiv: 1808.08967 ] [INSPIRE].

[15] Y. Akrami, R. Kallosh, A. Linde and V. Vardanyan, The landscape, the swampland and the era of precision cosmology, Fortsch. Phys. 67 (2019) 1800075 [arXiv: 1808. 09440] [INSPIRE].

[16] S. Kachru and S.P. Trivedi, A comment on effective field theories of flux vacua, Fortsch. Phys. 67 (2019) 1800086 [arXiv: 1808.08971] [INSPIRE].

[17] A. Hebecker and T. Wrase, The asymptotic dS swampland conjecture - A simplified derivation and a potential loophole, Fortsch. Phys. 67 (2019) 1800097 [arXiv:1810.08182] [INSPIRE].

[18] J.P. Conlon, The de Sitter swampland conjecture and supersymmetric AdS vacua, Int. J. Mod. Phys. A 33 (2018) 1850178 [arXiv: 1808.05040] [INSPIRE].

[19] H. Murayama, M. Yamazaki and T.T. Yanagida, Do we live in the swampland?, JHEP 12 (2018) 032 [arXiv: 1809.00478] [INSPIRE].

[20] J. Blåbäck, U. Danielsson and G. Dibitetto, A new light on the darkest corner of the landscape, arXiv: 1810.11365 [INSPIRE].

[21] S. Tsujikawa, Quintessence: a review, Class. Quant. Grav. 30 (2013) 214003 [arXiv: 1304.1961] [INSPIRE]. 
[22] P. Agrawal, G. Obied, P.J. Steinhardt and C. Vafa, On the cosmological implications of the string swampland, Phys. Lett. B 784 (2018) 271 [arXiv:1806.09718] [INSPIRE].

[23] A. Ashoorioon, Rescuing single field inflation from the swampland, Phys. Lett. B 790 (2019) 568 [arXiv: 1810.04001 ] [INSPIRE].

[24] M. Motaharfar, V. Kamali and R.O. Ramos, Warm inflation as a way out of the swampland, Phys. Rev. D 99 (2019) 063513 [arXiv:1810.02816] [InSPIRE].

[25] K. Dimopoulos, Steep eternal inflation and the swampland, Phys. Rev. D 98 (2018) 123516 [arXiv: 1810.03438] [INSPIRE].

[26] C.-M. Lin, K.-W. Ng and K. Cheung, Chaotic inflation on the brane and the swampland criteria, arXiv:1810.01644 [INSPIRE].

[27] S. Das, Note on single-field inflation and the swampland criteria, Phys. Rev. D 99 (2019) 083510 [arXiv: 1809.03962] [INSPIRE].

[28] S. Brahma and M. Wali Hossain, Avoiding the string swampland in single-field inflation: Excited initial states, JHEP 03 (2019) 006 [arXiv: 1809.01277] [INSPIRE].

[29] C. Damian and O. Loaiza-Brito, Two-field axion inflation and the swampland constraint in the flux-scaling scenario, Fortsch. Phys. 67 (2019) 1800072 [arXiv:1808.03397] [INSPIRE].

[30] H. Matsui and F. Takahashi, Eternal inflation and swampland conjectures, Phys. Rev. D 99 (2019) 023533 [arXiv:1807.11938] [INSPIRE].

[31] A. Kehagias and A. Riotto, A note on Inflation and the Swampland, Fortsch. Phys. 66 (2018) 1800052 [arXiv: 1807.05445] [INSPIRE].

[32] A. Achúcarro and G.A. Palma, The string swampland constraints require multi-field inflation, JCAP 02 (2019) 041 [arXiv: 1807.04390] [INSPIRE].

[33] J.-L. Lehners, Small-field and scale-free: inflation and ekpyrosis at their extremes, JCAP 11 (2018) 001 [arXiv: 1807.05240] [INSPIRE].

[34] C.-M. Lin, Type I hilltop inflation and the refined swampland criteria, Phys. Rev. D 99 (2019) 023519 [arXiv:1810.11992] [INSPIRE].

[35] S.C. Park, Minimal gauge inflation and the refined Swampland conjecture, JCAP 01 (2019) 053 [arXiv: 1810 . 11279] [INSPIRE].

[36] I. Ben-Dayan, Draining the swampland, Phys. Rev. D 99 (2019) 101301 [arXiv:1808.01615] [INSPIRE].

[37] C.-I. Chiang and H. Murayama, Building supergravity quintessence model, arXiv: 1808.02279 [INSPIRE].

[38] S.-J. Wang, Electroweak relaxation of cosmological hierarchy, Phys. Rev. D 99 (2019) 023529 [arXiv: 1810.06445] [INSPIRE].

[39] G. D'Amico, N. Kaloper and A. Lawrence, Strongly coupled quintessence, arXiv:1809.05109 [INSPIRE].

[40] C. Han, S. Pi and M. Sasaki, Quintessence saves Higgs instability, Phys. Lett. B 791 (2019) 314 [arXiv: 1809.05507] [INSPIRE].

[41] Y. Olguin-Trejo, S.L. Parameswaran, G. Tasinato and I. Zavala, Runaway quintessence, out of the swampland, JCAP 01 (2019) 031 [arXiv: 1810.08634] [INSPIRE]. 
[42] F. Denef, A. Hebecker and T. Wrase, De Sitter swampland conjecture and the Higgs potential, Phys. Rev. D 98 (2018) 086004 [arXiv: 1807.06581] [INSPIRE].

[43] K. Hamaguchi, M. Ibe and T. Moroi, The swampland conjecture and the Higgs expectation value, JHEP 12 (2018) 023 [arXiv: 1810.02095] [INSPIRE].

[44] G. Dvali and C. Gomez, On exclusion of positive cosmological constant, Fortsch. Phys. 67 (2019) 1800092 [arXiv: 1806.10877] [INSPIRE].

[45] D. Andriot, On the de Sitter swampland criterion, Phys. Lett. B 785 (2018) 570 [arXiv: 1806.10999] [INSPIRE].

[46] S.K. Garg and C. Krishnan, Bounds on slow roll and the de Sitter swampland, arXiv: 1807.05193 [INSPIRE].

[47] H. Ooguri, E. Palti, G. Shiu and C. Vafa, Distance and de Sitter conjectures on the swampland, Phys. Lett. B 788 (2019) 180 [arXiv:1810.05506] [InSPIRE].

[48] R. Bousso, A covariant entropy conjecture, JHEP 07 (1999) 004 [hep-th/9905177] [INSPIRE].

[49] C. Roupec and T. Wrase, De Sitter extrema and the swampland, Fortsch. Phys. 67 (2019) 1800082 [arXiv: 1807.09538 ] [INSPIRE].

[50] C. Caviezel, T. Wrase and M. Zagermann, Moduli stabilization and cosmology of type IIB on SU(2)-structure orientifolds, JHEP 04 (2010) 011 [arXiv:0912.3287] [INSPIRE].

[51] R. Flauger, S. Paban, D. Robbins and T. Wrase, Searching for slow-roll moduli inflation in massive type IIA supergravity with metric fluxes, Phys. Rev. D 79 (2009) 086011 [arXiv:0812.3886] [INSPIRE].

[52] Planck collaboration, Planck 2018 results. X. Constraints on inflation, arXiv:1807.06211 [INSPIRE].

[53] D.M. Scolnic et al., The complete light-curve sample of spectroscopically confirmed SNe Ia from Pan-STARRS1 and cosmological constraints from the combined pantheon sample, Astrophys. J. 859 (2018) 101 [arXiv:1710.00845] [INSPIRE].

[54] Planck collaboration, Planck 2015 results. XIII. Cosmological parameters, Astron. Astrophys. 594 (2016) A13 [arXiv:1502.01589] [INSPIRE].

[55] H. Fukuda, R. Saito, S. Shirai and M. Yamazaki, Phenomenological consequences of the refined swampland conjecture, Phys. Rev. D 99 (2019) 083520 [arXiv:1810.06532] [INSPIRE].

[56] S. Das, Warm inflation in the light of swampland criteria, Phys. Rev. D 99 (2019) 063514 [arXiv: 1810.05038] [INSPIRE]. 